\title{
Virtual monoenergetic images from photon-counting spectral computed tomography to assess knee osteoarthritis
}

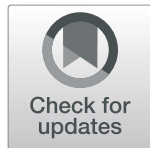

\author{
Christine Chappard ${ }^{1 *}$ (D), Juan Abascal ${ }^{2}$, Cécile Olivier² ${ }^{2}$ Salim Si-Mohamed² ${ }^{2}$ Loic Boussel², Jean Baptiste Piala², \\ Philippe Douek ${ }^{2}$ and Francoise Peyrin ${ }^{2}$
}

\begin{abstract}
Background: Dual-energy computed tomography has shown a great interest for musculoskeletal pathologies. Photon-counting spectral computed tomography (PCSCT) can acquire data in multiple energy bins with the potential to increase contrast, especially for soft tissues. Our objectives were to assess the value of PCSST to characterise cartilage and to extract quantitative measures of subchondral bone integrity.
\end{abstract}

Methods: Seven excised human knees ( 3 males and 4 females; 4 normal and 3 with osteoarthritis; age $80.6 \pm 14$ years, mean \pm standard deviation) were scanned using a clinical PCSCT prototype scanner. Tomographic image reconstruction was performed after Compton/photoelectric decomposition. Virtual monoenergetic images were generated from $40 \mathrm{keV}$ to $110 \mathrm{keV}$ every $10 \mathrm{keV}$ (cubic voxel size $250 \times 250 \times 250 \mathrm{\mu m}^{3}$ ). After selecting an optimal virtual monoenergetic image, we analysed the grey level histograms of different tissues and extracted quantitative measurements on bone cysts.

Results: The optimal monoenergetic images were obtained for $60 \mathrm{keV}$ and $70 \mathrm{keV}$. Visual inspection revealed that these images provide sufficient spatial resolution and soft-tissue contrast to characterise surfaces, disruption, calcification of cartilage, bone osteophytes, and bone cysts. Analysis of attenuation versus energy revealed different energy fingerprint according to tissues. The volumes and numbers of bone cyst were quantified.

Conclusions: Virtual monoenergetic images may provide direct visualisation of both cartilage and bone details. Thus, unenhanced PCSCT appears to be a new modality for characterising the knee joint with the potential to increase the diagnostic capability of computed tomography for joint diseases and osteoarthritis.

Keywords: Bone cysts, Cartilage, Osteophyte, Osteoarthritis (knee), Tomography (X-ray computed)

\section{Key points}

- Photon-counting spectral compute tomography (PCSCT) is a new tool to explore joints with high spatial resolution.

- Virtual monoenergetic images at $60 \mathrm{keV}$ and $70 \mathrm{keV}$ provided sufficient soft-tissue contrast to

\footnotetext{
* Correspondence: christine.chappard@inserm.fr

'B3OA, CNRS UMR 7052, U 1271 Inserm, University of Paris, Paris, France Full list of author information is available at the end of the article
}

characterise surfaces, disruption, calcification of cartilage, bone osteophytes, and bone cysts.

- On the PCSCT virtual monoenergetic images at 60 $\mathrm{keV}$, volume and density of bone cysts can be quantified semiautomatically.

\section{Background \\ Osteoarthritis $(\mathrm{OA})$ is a chronic inflammatory joint dis- order characterised by cartilage loss, abnormal subchon- dral bone, osteophyte formation, degeneration of ligaments, meniscus, and hypertrophy of the capsule [1]. The damage to the cartilage is typically characterised by}

\section{Springer Open}


fissures in the superficial layers, which gradually extend to deeper zones, and finally full cartilage loss [2]. It is essential to detect early changes during the reversible phase of the disease. Progresses in OA management require the development of noninvasive diagnostic methods that can be used to quantify changes in the cartilage and subchondral bone [3].

Imaging methods used for diagnosis of OA are usually conventional radiography and magnetic resonance imaging, the latter being classically used in clinical routine to visualise joint effusion, cartilage, ligaments, tendons, meniscus, osteophytes, and bone marrow oedema.

Computed tomography (CT) is not ideally suited to the observation of soft tissue like cartilage, however when combined with intra-articular application of a contrast agent, it can be used to investigate the cartilage surface. Moreover, CT has been proposed for chondrocalcinosis diagnosis [4], and for quantification of both volume and number of subchondral bone cysts [5].

Dual-energy CT, combining measurements from two energy spectra has been shown for its use in musculoskeletal imaging particularly in the detection of gout [6]. However, a recent publication showed that this technique does not bring more information than conventional CT for calcium pyrophosphate deposition [7], and to the best of our knowledge, it is not used for cartilage analysis. Today, the new generations of photon-counting spectral CT (PCSCT) scanners include energydiscriminating photon-counting detectors (PCDs) that can simultaneously count photons and resolve their energy $[8,9]$ conversely to conventional CT scanners energy-integrating detectors. With such systems, it is possible to reconstruct different types of images like material decomposition images or virtual monoenergetic images. Another advantage of this new detector is the improved signal-to-noise ratio (SNR), due to the exclusion of electronic noise [10]. With such additional information, PCSCT is expected to surpass conventional CT and open new possibilities in medical diagnosis.

PCSCT is ideal for material decomposition imaging, as for K-edge imaging, which uses the discontinuity at diagnostic energies of the linear attenuation coefficient of high-Z element-based contrast agents, such as gadolinium, gold, and bismuth $[11,12]$. Indeed, PCSCT combined with one or several contrast agents has been proposed for diverse applications. These included tracking and monitoring of the biodistribution of gold nanoparticles in vivo [13], determination of contrast agent concentrations in the liver [14], and evaluation of the risk of breast cancer [15]. PCSCT might also be used to quantify calcium content (e.g., in bone, teeth, kidney stones, coronary plaques) and to discriminate between different calcium crystals [16, 17]. In addition to material decomposition and K-edge imaging, PCSCT offers the possibility to obtain virtual monoenergetic images, with the potential to increase the contrast especially for soft tissues [18]. This feature and the reduced detector pixel size provided by PCD makes PCSCT a new candidate for medical applications where resolution and soft tissue contrast are critical. Considering its applications to OA to date, PCSCT coupled to an iodine contrast agent has only been considered in one study, for measuring proteoglycan content in cartilage in knee specimens [19]. Thus, there are no current methods that have sufficiently high resolution and image quality to visualise the internal structures of the bone, the meniscus, and cartilage details at the same time [20].

Thus, the aim of this study was to investigate the feasibility of PCSCT for assessment of joint integrity without the need for a contrast agent. Our first aim is to evaluate whether virtual monoenergetic reconstructions produced sufficient spatial resolution and soft tissue contrast to visualise both cartilage and bone with enough details without contrast agent. Our second aim is to evaluate the quality of the images produced is sufficient to perform quantitative analysis.

\section{Methods}

\section{Sample description}

Seven knee specimens from 3 males and 4 females, aged $80.6 \pm 14$ years (mean \pm standard deviation) were obtained from the Institut d'Anatomie Paris (France). The collection of these human tissue specimens was conducted according to the relevant protocols established by the Human Ethics Committee from the Institute of Medical Research. No additional information was available regarding cause of death, previous illnesses, or any medical treatments of these subjects except for an absence of hepatitis and human immunodeficiency virus. The protocol was approved by the French Ministry of Higher Education and Research (CODECOH number DC-2019-3422). After soft tissue removal, the knee specimens were stored at $-20{ }^{\circ} \mathrm{C}$.

\section{Data acquisition and image reconstruction}

The knee specimens were imaged using a clinical PCSC $\mathrm{T}$ prototype system (Philips Healthcare, Amsterdam, Netherland) installed at CERMEP, Lyon. This is a modified clinical system that is equipped with a conventional $\mathrm{X}$-ray tube that can be set to tube voltages from 80 to $120 \mathrm{kVp}$, and tube currents between $10 \mathrm{~mA}$ and 500 $\mathrm{mA}$; it was set at $120 \mathrm{kVp}$ and $100 \mathrm{~mA}$ in the present study. The tube filtration absorbs low-energy X-rays, so the final spectrum ranges from $30 \mathrm{keV}$ to $120 \mathrm{keV}$. The system is based on PCDs of 2-mm-thick cadmium zinc telluride, with a pixel pitch of $270 \times 270 \mu \mathrm{m}^{2}$ at the isocenter, and coupled with application-specific integrated circuits (ChromAIX2, Philips Research Europe, Aachen, 
Germany) that operate in single-photon-counting mode with energy discrimination [21]. The acquisition time was less than $5 \mathrm{~min}$. The PCDs allow up to five consecutive energy thresholds between $30 \mathrm{keV}$ and $120 \mathrm{keV}$, which were set in the present study at $30,51,62,72$, and $81 \mathrm{keV}$. The acquisition field of view was $500 \mathrm{~mm}$ inplane, with a $z$-coverage of $17.5 \mathrm{~mm}$ in the scanner isocenter. Axial scans were performed over $360^{\circ}$ with 2,400 projections per rotation, on a grid of $64 \times 1,848$ pixels.

Fifty stacks of eight slices were acquired to cover the entire knee specimens (height, $10 \mathrm{~cm}$ ). After data acquisition, the projections in the different energy bins were decomposed on a Compton/photoelectric basis using the maximum-likelihood method [11]. Then, the decomposed Compton/photoelectric sinograms (Radon transform) were reconstructed using filtered back-projection. The whole reconstructed images were made of $640 \times$ $640 \times 400$ voxels, with a voxel size of $250 \times 250 \times 250$ $\mu \mathrm{m}^{3}$ with a reconstructed scan field of view about $160 \times$ $160 \times 100 \mathrm{~mm}^{3}$. Seven virtual monoenergetic images from $40 \mathrm{keV}$ to $110 \mathrm{keV}$ were then computed from the linear combination of the reconstructed Compton/ photoelectric images and expressed in Hounsfield units (HU) units. In addition, the conventional HU image obtained by combining all of the bins together was computed.

For comparison, we used a standard HR-pQCT imaging protocol (Scanco Wangen-Brüttisellen, Switzerland) with voxel size of $82 \mu \mathrm{m}$ for all specimens, and synchrotron radiation monochromatic CT at 55 $\mathrm{KeV}$ (European Synchrotron Radiation Facility, Grenoble beamline ID 17) with a voxel size at $45 \mu \mathrm{m}$ for 5 specimens. The Kellgren-Lawrence classification [22] was performed on the HR-pQCT images.

\section{Image analysis}

First, we investigated the multienergy feature of PCSCT. For this purpose, we analysed the monoenergetic images and compared them to the conventional HU images to select an optimal monoenergetic image for cartilage assessment. This required selection of the energy that led to the best characterisation of the cartilage in terms of noise and contrast-to-noise ratio (CNR). The noise was computed as the standard deviation (SD) for a manually selected circular region of interest with radius of 4 pixels in a homogeneous part within the cartilage. The CNR was computed as the difference for the grey levels along a line inside the cartilage and a line inside the joint space outside the meniscus divided by the SD previously computed. The monoenergetic approach allows to retrieve the information that links the X-ray attenuation to the type of material crossed and the energy. We analysed the attenuation variation of cartilage, of both trabecular and cortical bone and soft tissue with energy for all knees.

The second part of the image analysis consisted of the qualitative assessment of $\mathrm{OA}$ from the monoenergetic images at the selected energy. For this, the feasibility of visual assessment of cartilage defects, cartilage calcification, bone cysts, and bone osteophytes was investigated by an expert radiologist who had been in practice for > 20 years (J.B.P.).

The third part concerned the extraction of quantitative parameters from the monoenergetic images at the selected energy. We analysed bone cysts observed in the monoenergetic images for the two severe OA knee specimens. To this aim, we performed the segmentation of the subchondral bone of the femur and patella in the femoro-patellar compartments to a total depth of 10 $\mathrm{mm}$ from the subchondral surface. The bone cysts were segmented using the commercially available Avizo 9.0 software (FEI Visualization Sciences Group, Burlington MA, Avizo v.9.0) with the semiautomatic region growing tool (Magic Wand). Here, a seed point was first defined by the operator, and all of the connected voxels with grey levels in a given tolerance range were selected. Any object with a volume $<20$ voxels $\left(0.31 \mathrm{~mm}^{3}\right)$ was considered as noise, and was removed from the final calculations. The bone cysts in the medial and lateral compartments were analysed separately. After segmentation, the following parameters were measured: number of cysts $/ \mathrm{mm}^{3}$, total cyst volume $\left(\mathrm{mm}^{3}\right)$, and maximum cyst volume $\left(\mathrm{mm}^{3}\right)$. Our quantitative analysis was exploratory, so not supported by statistical analysis.

\section{Results}

\section{Multienergy imaging}

Details from one normal specimen (sample 3) are presented here for the reconstructed images from the decomposed photoelectric and Compton images, the conventional $\mathrm{HU}$ image (from merging of all of the energy bins), and the virtual monoenergetic images, from $40 \mathrm{keV}$ to $110 \mathrm{keV}$ (Fig. 1). We note that the cartilage is visible in the Compton and conventional HU images, and in the virtual monoenergetic images above $50 \mathrm{keV}$. High-energy monoenergetic images above $80 \mathrm{KeV}$ appeared to provide not only improved contrast, but also higher noise. Among all of these monoenergetic images, those for $60 \mathrm{keV}$ and $70 \mathrm{keV}$ appeared less noisy and with greater image quality than the Compton and conventional HU images.

The noise was computed as the SD inside the cartilage, and the CNR as the differences for the grey levels between the cartilage and the surrounding soft tissue for all of the monoenergetic images and the conventional images (Fig. 2). Among the monoenergetic images, 60 $\mathrm{keV}$ showed the lowest noise levels and the highest 


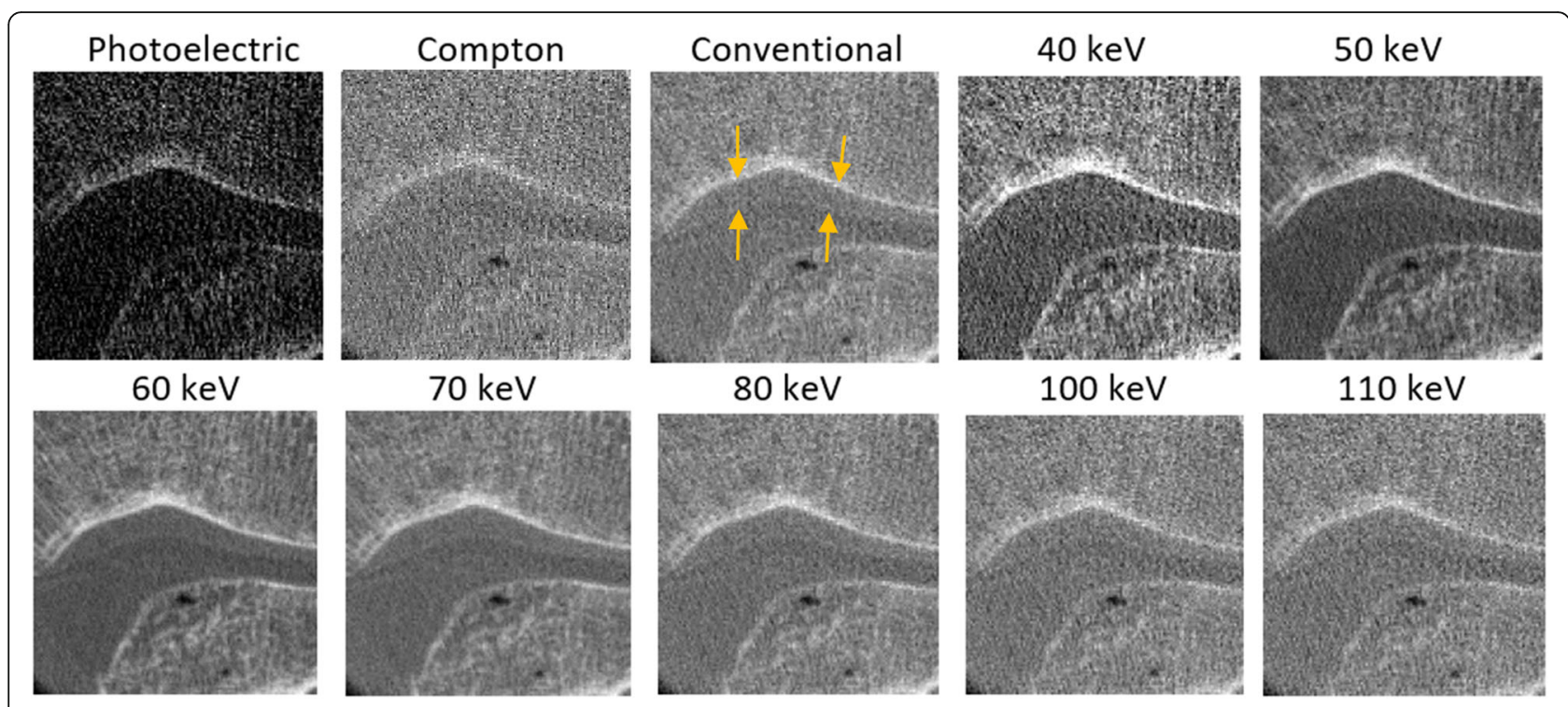

Fig. 1 Details of the reconstructed images for the decomposed materials (photoelectric and Compton effects), conventional image (merging all energy bins), and virtual monoenergetic images (as indicated) for one normal specimen. Orange arrows, upper and lower surfaces of cartilage
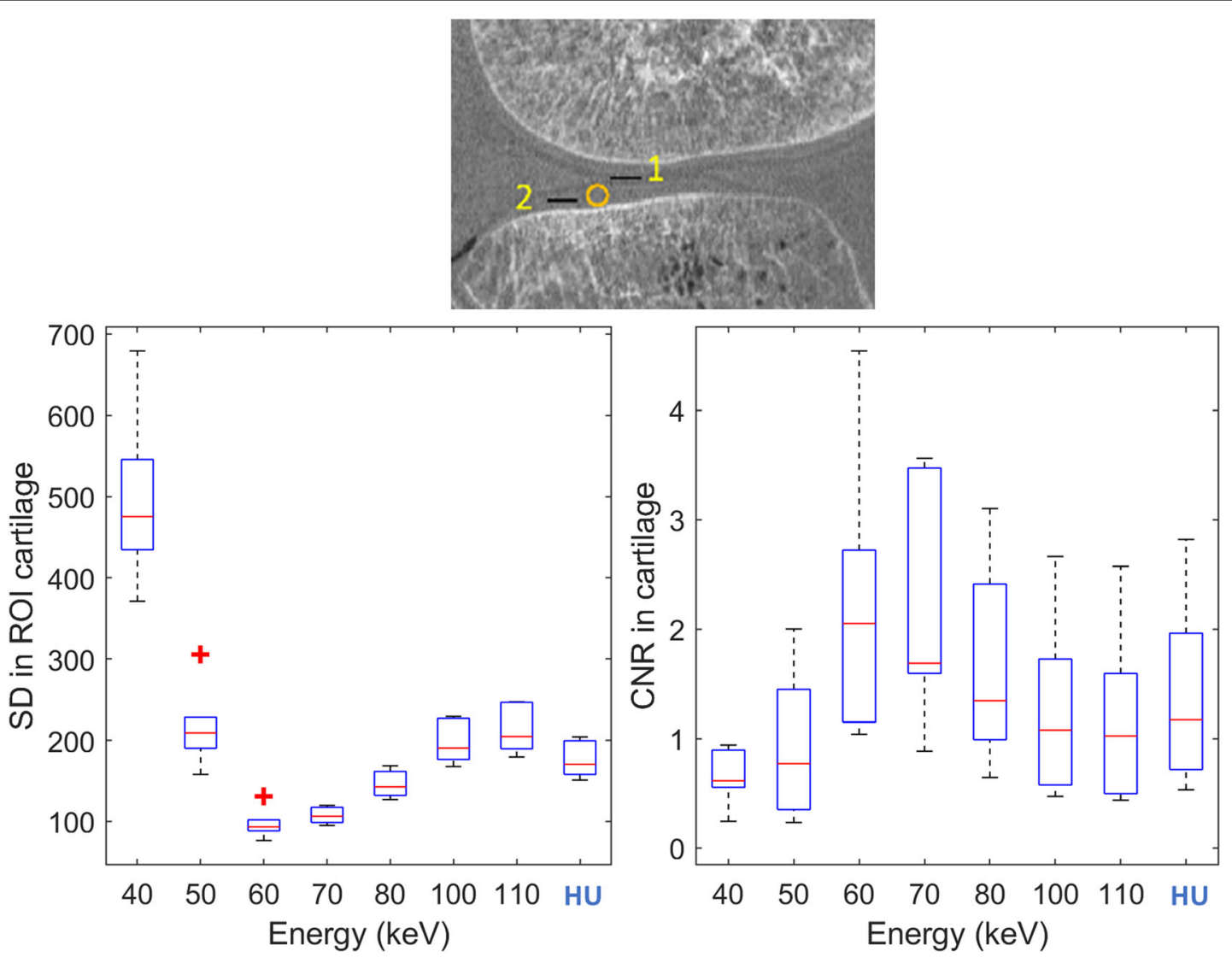

Fig. 2. Noise standard deviation (SD) (left) and contrast-to-noise ratio (CNR) (right) for the cartilage for all of the monoenergetic images (40-110 $\mathrm{keV}$ ) and the conventional image (HU). Plots display median and confidence intervals across the seven samples, with red crosses to indicate outliers. Top: Region-of-interest masks used for the analysis. Noise is computed as the SD within the circular region in the cartilage. CNR is computed as the contrast given by black lines 1 and 2 divided by the SD 
CNR, where the noise was a little lower than that at 70 $\mathrm{keV}$. Adopting the conventional $\mathrm{HU}$ image as reference, the $60 \mathrm{keV}$ monoenergetic image led to a $45 \%$ reduction in noise and $75 \%$ increase in CNR, which is relevant for good visualisation of cartilage.

Cartilage, soft tissue surrounding bone, and the different bone segments showed large variations in attenuation versus energy (Fig. 3). At $60 \mathrm{keV}$, the attenuation values ranged from 1,551 to $1,581 \mathrm{HU}$ for cartilage and from 1,447 to $1,503 \mathrm{HU}$ for soft tissue.

\section{Qualitative assessments of monoenergetic images}

The results of application of the Kellgren-Lawrence classification on the HR-pQCT images are described in Table 1. Cartilage defects qualified by a $75 \%$ local cartilage height loss, cartilage calcification, bone cysts are visible on the $60 \mathrm{keV}$ monoenergetic images (Fig. 4). Bone osteophytes for subjects across the different levels of OA are pointed out (Fig. 4). Table 1 gives the clinical descriptions of all studied knee specimens relative to the cartilage aspects, osteophytes and subchondral bone cysts.

Figure 5 shows the three-dimensional displays of the $60 \mathrm{keV}$ monoenergetic images of two selected samples: left, OA (sample 2); right, normal (sample 3). The top images include the femur, the tibia, and the patella. In the bottom images, the patella has been numerically removed to visualise the cartilage surface. Indeed, cartilage surface defects and calcifications can be seen for the OA specimen (left), with no defect apparent for the normal specimen (right). Subchondral bone cysts segmentation of the OA specimen in the femur (sample 2) is also displayed on Fig. 5.

PCSCT allowed the visualisation of the cartilage with a quality approaching synchrotron radiation CT especially for the border of cartilage with joint space (Fig. 6). On the contrary, cartilage was not visible on conventional CT such as HR-pQCT with energy integrating detectors, even with high spatial resolution.

\section{Quantitative analysis of selected monoenergetic images}

Bone cysts were found in two OA specimens (sample 1 and sample 2), and were segmented for both the femur and patella in a region up to $10 \mathrm{~mm}$ from the cartilage, to measure the quantitative parameters. The number and total volume of the bone cysts, and their maximal volume for each knee are given in Table 2. A threedimensional display of the segmented bone cysts is provided in Fig. 5 (red) in sample 2.

\section{Discussion}

Virtual monoenergetic images can provide direct visualisation of the details of the cartilage and bone on the same image. To the best of our knowledge, this is the first study to investigate PCSCT for OA without the need for any contrast agent. We have shown that the 60 $\mathrm{keV}$ and $70 \mathrm{keV}$ monoenergetic images are optimal in terms of noise and CNR, respectively, and that these provide superior image quality compared to the $\mathrm{HU}$ images.

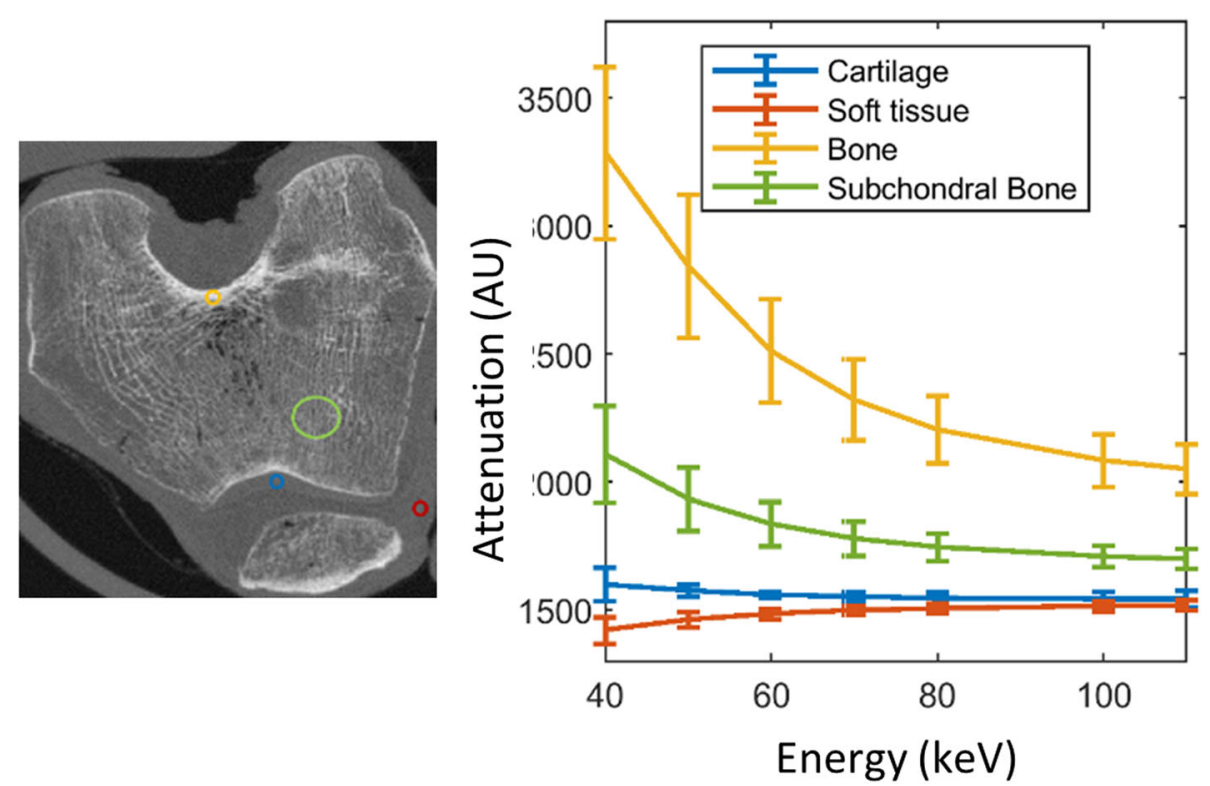

Fig. 3 Right: Energy fingerprints of the different joint tissues computed from the virtual monoenergetic images. Attenuation versus energy for the regions of interest shown in the left panel: patella cartilage (blue), soft tissue different from cartilage (red), cortical bone (yellow), and subchondral bone (green). Plot shows mean and standard deviation across subjects for the selected regions of interest 
Table 1 Clinical descriptions of the knee specimens in terms of cartilage, osteophytes, and bone cyts

\begin{tabular}{|c|c|c|c|c|c|c|c|}
\hline \multirow{2}{*}{$\begin{array}{l}\text { Sample } \\
\text { Cartilage }\end{array}$} & \multirow[t]{2}{*}{ Sex } & \multirow[t]{2}{*}{ Age } & \multirow[t]{2}{*}{ KL } & \multicolumn{2}{|l|}{ Lateral } & \multicolumn{2}{|l|}{ Medial } \\
\hline & & & & Femur & Patella & Femur & Patella \\
\hline 1 & $\mathrm{~F}$ & 89 & 4 & Thin, defects & $\begin{array}{l}\text { Thin, defects } \\
\text { calcifications+ }\end{array}$ & Thin, defects & Thin, defects \\
\hline 2 & M & 89 & 3 & $\begin{array}{l}\text { Thin, irregular } \\
\text { calcifications+ }\end{array}$ & $\begin{array}{l}\text { Thin, irregular } \\
\text { calcifications+ }\end{array}$ & $\begin{array}{l}\text { Thin, defects } \\
\text { calcifications+ }\end{array}$ & $\begin{array}{l}\text { Thin, irregular } \\
\text { calcifications+ }\end{array}$ \\
\hline 3 & M & 59 & 1 & Normal, smooth & Normal, smooth & Normal, defects & Normal, smooth \\
\hline 4 & $\mathrm{~F}$ & 90 & 0 & Normal, smooth & Thin, defects & Normal, smooth & $\begin{array}{l}\text { Normal, irregular } \\
\text { calcifications+ }\end{array}$ \\
\hline 5 & $\mathrm{~F}$ & 81 & 0 & Normal, smooth & $\begin{array}{l}\text { Normal, smooth } \\
\text { calcifications+ }\end{array}$ & $\begin{array}{l}\text { Normal, smooth } \\
\text { calcifications+ }\end{array}$ & $\begin{array}{l}\text { Normal, smooth } \\
\text { Calcifications+ }\end{array}$ \\
\hline 6 & $\mathrm{~F}$ & 94 & 1 & Normal, irregular & Normal, irregular & Normal, smooth & Normal, smooth \\
\hline 7 & M & 63 & 2 & Thin, defects & Thin, defects & Normal, defects & Thin, defects \\
\hline \multicolumn{8}{|c|}{ Subchondral bone cysts } \\
\hline 1 & & & & Large size, numerous & Large size, numerous & Large size numerous & Large size, numerous \\
\hline 2 & & & & $\begin{array}{l}\text { Middle size } \\
\text { few }\end{array}$ & $\begin{array}{l}\text { Small size } \\
\text { numerous }\end{array}$ & $\begin{array}{l}\text { Middle size } \\
\text { few }\end{array}$ & - \\
\hline 3 & & & & - & - & - & - \\
\hline 4 & & & & $\begin{array}{l}\text { Middle size } \\
\text { numerous }\end{array}$ & $\begin{array}{l}\text { Large size } \\
\text { numerous }\end{array}$ & - & $\begin{array}{l}\text { Middle size } \\
\text { few }\end{array}$ \\
\hline 5 & & & & - & - & - & - \\
\hline 6 & & & & - & - & - & - \\
\hline 7 & & & & $\begin{array}{l}\text { Small size } \\
\text { few }\end{array}$ & $\begin{array}{l}\text { Small size } \\
\text { few }\end{array}$ & - & - \\
\hline \multicolumn{8}{|c|}{ Osteophytes } \\
\hline 1 & & & & Large size & Small size & Large size & Large size \\
\hline 2 & & & & - & Small size & Small size & - \\
\hline 3 & & & & - & - & - & - \\
\hline 4 & & & & - & - & - & - \\
\hline 5 & & & & - & - & - & - \\
\hline 6 & & & & - & - & - & - \\
\hline 7 & & & & Small size & Small size & Small size & Small size \\
\hline
\end{tabular}

In a previous PCSCT study performed in vivo for the abdomen [18], $72 \mathrm{keV}$ monoenergetic images showed improved contrast and SNR compared to conventional images with similar attenuation patterns. In the present study, the monoenergetic images in the range from 60 $\mathrm{keV}$ to $80 \mathrm{keV}$ had lower noise than the conventional images, with the $60 \mathrm{keV}$ and $70 \mathrm{keV}$ monoenergetic images showing similar noise levels, although the lowest noise overall was for $60 \mathrm{keV}$. These data confirm the improvements in the SNR demonstrated in a previous simulated study that used a cadmium zinc telluride PCD in comparison to conventional detectors [21]. Indeed, the intrinsic qualities of PCDs can explain SNR and CNR increases: first, the detector counts the number of pulses greater than the preset threshold, which consequently eliminates the electronic noise; secondly, high energy photons contribute more than low energy photons in conventional detectors, which consequently decreases the CNR. In contrast, for PCDs, the equally weighted energy photons have positive effects on the contrast [9]. We hypothesise that the higher resolution and higher CNR in soft tissue for PCSCT with respect to standard CT will lead to superior diagnosis potential for applications for which resolution and contrast are critical. In addition, another major advantage of virtual monoenergetic images is that they provide quantitative attenuation measurements and reduce beam hardening artifacts [23]. Finally, one of the major advantages of PCD detectors is the patient's radiation dose reduction. Indeed, based on an American College of Radiology accredited phantom, it was demonstrated that image quality can be maintained with a reduction in the dose of 40 to $60 \%$ [24].

Different joint tissues showed specific energy fingerprints. In particular, no overlap was seen between the 


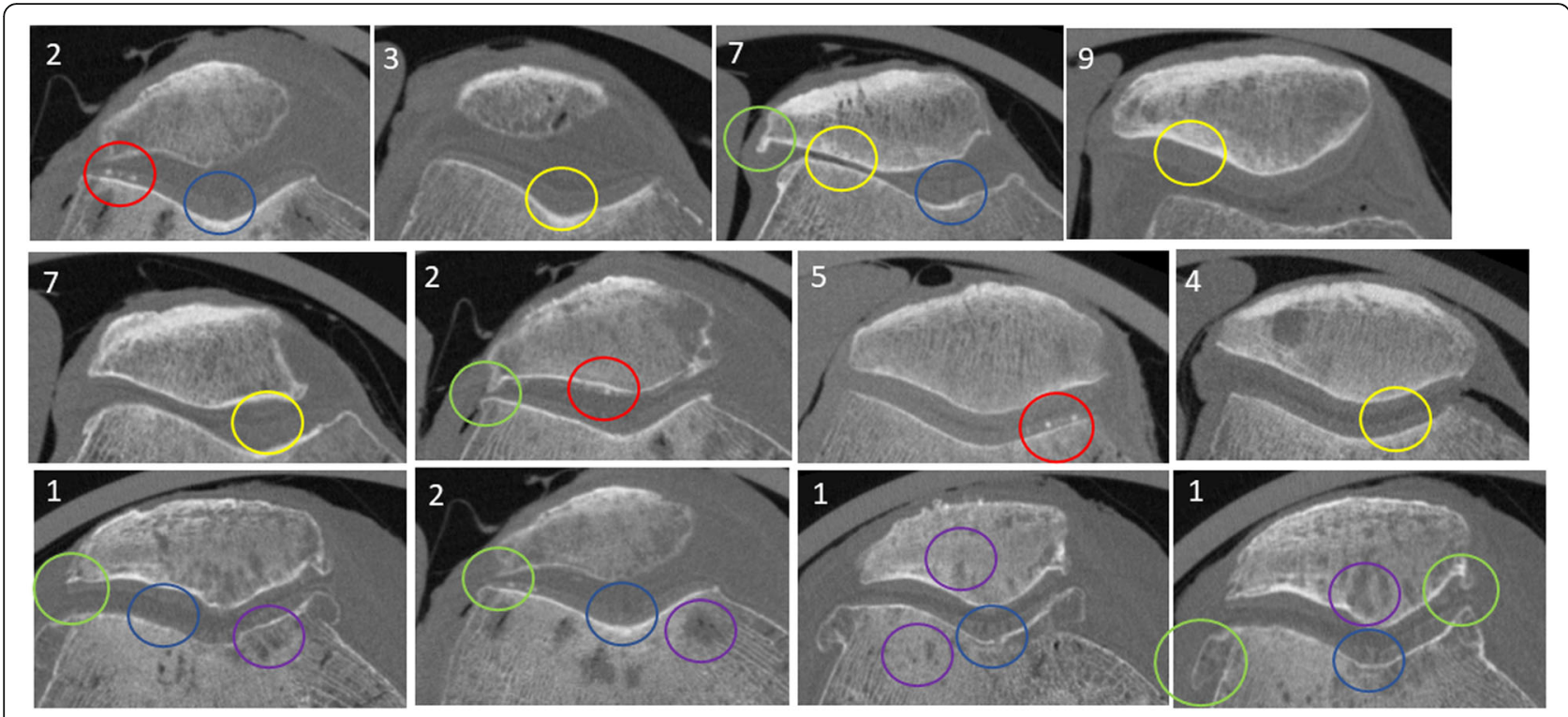

Fig. 4 Transversal views of the selected $60 \mathrm{keV}$ monoenergetic images for six specimens, the specimens 1 and 2 are severe osteoarthritis and sample 7 moderate osteoarthritis with numbers corresponding to samples. Red circle, cartilage calcifications; yellow circle, cartilage with different thicknesses; blue circle, cartilage defects; purple circle, bone cysts of various size; green circle: osteophytes of various size

cartilage and the soft tissue surrounding the cartilage. The analysis of the histograms showed that subjects with cartilage defects are shifted in the lower part of the histograms and on the contrary the presence of calcifications shifted the histogram in the high part.

Indeed, it is possible to discriminate calcifications in cartilage and the meniscus. CT has been proposed to evaluate calcium deposition, which is especially useful in the early stages of OA [24]. Using a preclinical scanner with high-resolution (i.e., $0.1 \mathrm{~mm}^{3}$ voxels) and low- energy monoenergetic images (i.e., $20-80 \mathrm{keV}$ ), it is possible to differentiate different calcium deposits in peripheral joints, such as calcium pyrophosphate and calcium apatite $[16,17]$ In the present study, PCSCT allowed the characterisation of bone cysts, which are related to mechanical stress in OA knees, and consequently this will be interesting to study further for better understanding of the OA process [25] and it will also help to better phenotype such OA patients [26] and cysts formation following cartilage repair [27]. Indeed, bone cysts

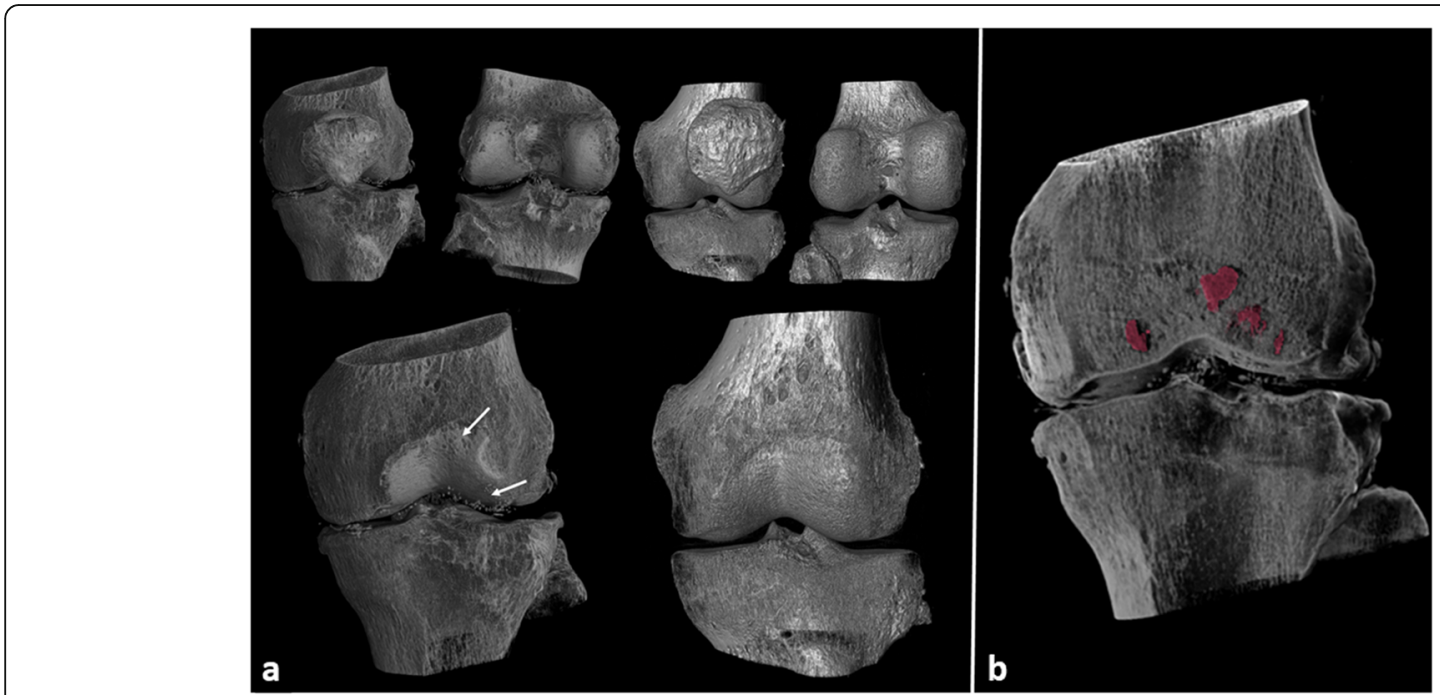

Fig. 5 Three-dimensional displays of the $60 \mathrm{keV}$ monoenergetic for two selected samples. a, left, OA (sample 2); a, right normal (sample 3). White arrows show cartilage defects (top) and calcifications (bottom) on OA. b lllustration of segmented bone cysts (in red) with the semiautomatic region growing tool in OA sample (sample 2) 


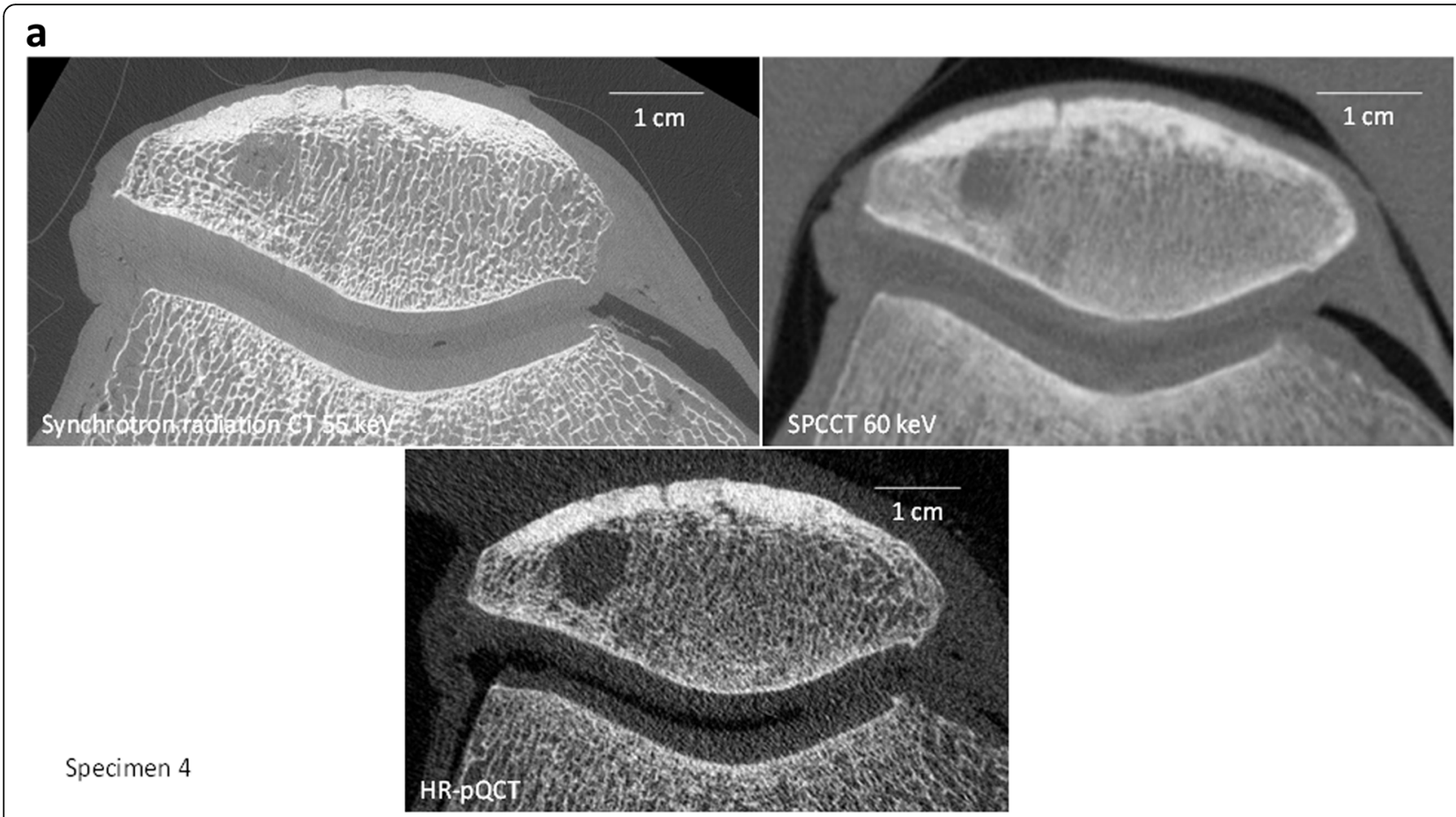

b
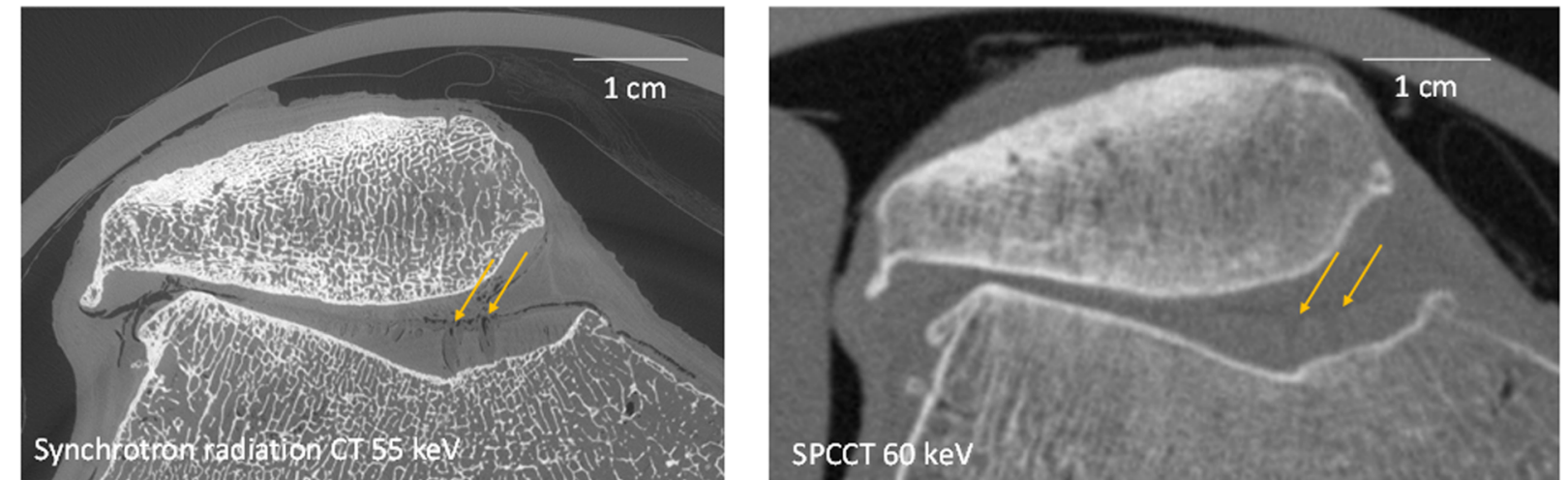

Specimen 7

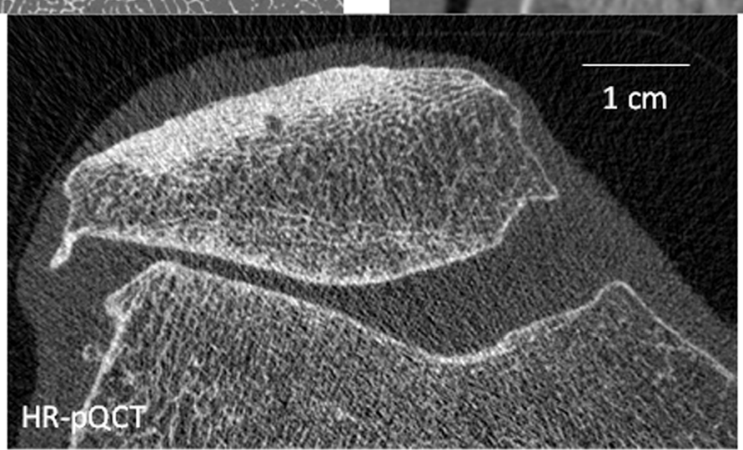

Fig. 6 Synchrotron radiation images at $55 \mathrm{keV}$, PCSCT monoenergetic images at $60 \mathrm{keV}$ and HR-pQCT images for 2 samples: a normal one (specimen 4); and an osteoarthritic one (specimen 7). The cartilage border with joint space is visible on the monoenergetic images based on synchrotron radiation, taken as references and the virtual PCSCT monoenergetic images. The yellow arrows correspond to cartilage defects 
Table 2 Quantitative analysis of the bone cysts for two of the specimens

\begin{tabular}{|c|c|c|c|c|c|}
\hline \multirow[t]{3}{*}{ Specimen } & \multirow[t]{3}{*}{ Site } & \multirow[t]{3}{*}{ Compartment } & \multicolumn{3}{|l|}{ Cysts } \\
\hline & & & \multirow[t]{2}{*}{ Number } & \multicolumn{2}{|c|}{ Volume $\left(\mathrm{mm}^{3}\right)$} \\
\hline & & & & Total & Maximum \\
\hline \multirow[t]{6}{*}{ Sample 1} & Femoral & Medial & 8 & 185.8 & 110.6 \\
\hline & & Lateral & 44 & 355 & 106.1 \\
\hline & & Total & 52 & 540.8 & 110.6 \\
\hline & Patellar & Medial & 10 & 105.1 & 31 \\
\hline & & Lateral & 12 & 371 & 204.9 \\
\hline & & Total & 22 & 476.1 & 204.9 \\
\hline \multirow[t]{6}{*}{ Sample 2} & Femoral & Medial & 3 & 241.8 & 109.5 \\
\hline & & Lateral & 4 & 223.2 & 125.5 \\
\hline & & Total & 7 & 465 & 125.5 \\
\hline & Patellar & Medial & 4 & 13.2 & 5.5 \\
\hline & & Lateral & 11 & 37.9 & 17.7 \\
\hline & & Total & 15 & 51.1 & 17.7 \\
\hline
\end{tabular}

quantification were rarely performed from standard CT [26] and one time from high-resolution peripheral CT [28].

The present study is subject to some limitations. The reconstructed images depend on the material decomposition and the postprocessing methods used. Material decomposition was carried out on a photoelectric and Compton basis. Other specific material basis, such as those based on bone and soft tissue, or even more general basis, are possible [29]. The investigation of other potentially improved basis might lead to further information with regard to the analysis of cartilage and calcifications. The material decomposition method used was based on conventional maximum likelihood, without regularisation in a pixel-bypixel manner [11]. More advances inverse methods including regularisation [30-33] or based on deep learning approaches [34-36] might lead to improvements here. The image post-processing here was specifically designed for knee images; it might be further improved [37]. Our quantitative analysis is exploratory at this level, to show the potential of PCSCT for OA.

However, more refined methods in combination with a larger number of samples might lead to new biomarkers that translate into more accurate and meaningful OA scoring, and consequently to improved diagnosis. Further experiments carried out in vivo and with more subjects are needed to validate these findings.

In conclusion, virtual monoenergetic can provide direct visualisation of both cartilage and bone integrity, which are essential for the characterisation of osteoarticular diseases that affect the whole joint, with this demonstration thus focused on OA. In addition, these data suggest that quantitative measurements of bone cysts in terms of numbers and size might lead to new biomarkers for better phenotyping of OA. The prototype is operational for clinical research, with further studies needed to validate these data on living patients.

\section{Abbreviations}

CNR: Contrast-to-noise ratio; HU: Hounsfield unit; OA: Osteoarthritis; PCD: Photon-counting detectors; PCSCT: Photon-counting spectral computed tomography; SD: Standard deviation; SNR: Signal-to-noise ratio

Authors' contributions

All authors read, edited, and approved the final version of the manuscript. CC carried out the study concept, design, data acquisition, analysis and interpretation, and manuscript preparation. JA contributed to data acquisition, data analysis, and manuscript preparation. CO contributed to data acquisition and analysis. SSM contributed to data acquisitions. LB contributed to data analysis. JBP carried out the CT examination. PD contributed to study concept, design, interpretation. FP carried out the study concept, design, data acquisition, analysis, and manuscript editing.

\section{Funding}

This project was supported by the ANR project SALTO (ANR-17-CE19-001101), within LabEx PRIMES (ANR-11-LABX-0063) of University de Lyon. It has received funding from the European Union Horizon 2020 Research and Innovation Program under Marie Sklodowska-Curie grant agreement $\mathrm{N}^{\circ}$ 701915. The project also received funding from the European Union Horizon 2020 Research and Innovation Program under grant agreement $N^{\circ} 668142$. This work was partly funded by France Life Imaging (grant ANR-11-INBS0006) from the French Investissements d'Avenir.

\section{Availability of data and materials}

The datasets used and/or analysed during the current study are available from the corresponding author on reasonable request.

\section{Declarations}

Ethics approval and consent to participate

The study was approved by the Ethics Committee of Paris University, Paris. The tissue donors or their legal guardians provided informed written consent to give their tissue for investigations, in accord with legal clauses.

\section{Consent for publication}

Not applicable

\section{Competing interests}

The authors of this manuscript declare no relationships with any companies whose products or services may be related to the subject matter of the article.

\section{Author details}

'B3OA, CNRS UMR 7052, U 1271 Inserm, University of Paris, Paris, France. 2University of Lyon, INSA-Lyon, CNRS, Inserm, CREATIS UMR 5220, U1206, Lyon, France.

Received: 27 September 2021 Accepted: 30 December 2021

Published online: 22 February 2022

\section{References}

1. Loeser RF, Goldring SR, Scanzello CR, Goldring MB (2012) Osteoarthritis: a disease of the joint as an organ. Arthritis Rheum 64:1697-1707. https://doi. org/10.1002/art.34453 Epub 2012 Mar 5

2. Thysen S, Luyten FP, Lories RJU (2015) Targets, models and challenges in osteoarthritis research. Dis Model Mech 8:17-30. https://doi.org/10.1242/ dmm.016881

3. Berenbaum F (2010) Targeted therapies in osteoarthritis: a systematic review of the trials on www.clinicaltrials.gov. Best Pract Res Clin Rheumatol 24:107119. https://doi.org/10.1016/j.berh.2009.08.007 
4. Magarelli N, Amelia R, Melillo N, Nasuto M, Cantatore F, Guglielmi G (2012) Imaging of chondrocalcinosis: calcium pyrophosphate dihydrate (CPPD) crystal deposition disease -- imaging of common sites of involvement. Clin Exp Rheumatol 30:118-125

5. Burnett WD, Kontulainen SA, McLennan CE et al (2019) Knee osteoarthritis patients with more subchondral cysts have altered tibial subchondral bone mineral density. BMC Musculoskelet Disord 20:14. https://doi.org/10.1186/ s12891-018-2388-9

6. Metzger SC, Koehm M, Wichmann JL, et al (2016) Dual-energy CT in patients with suspected gouty arthritis: effects on treatment regimen and clinical outcome. Acad Radiol 23:267-272. https://doi.org/10.1016/j.acra.201 5.10.019

7. Budzik JF, Marzin C, Legrand J, Norberciak L, Becce F, Pascart T (2021) Can dual-energy computed tomography be used to identify early calcium crystal deposition in the knees of patients with calcium pyrophosphate deposition. Arthritis Rheumatol 73:687-692. https://doi.org/10.1002/art.41 569

8. Taguchi K, Iwanczyk JS (2013) Vision 20/20: Single photon counting X-ray detectors in medical imaging. Med Phys 40:100901. https://doi.org/10.111 8/1.4820371

9. Si-Mohamed S, Bar-Ness D, Sigovan M, et al (2018) Multicolour imaging with spectral photon-counting CT: a phantom study. Eur Radiol Exp 2:34. https://doi.org/10.1186/s41747-018-0063-4

10. Willemink MJ, Persson M, Pourmorteza A, Pelc NJ, Fleischmann D (2018) Photon-counting CT: technical principles and clinical prospects. Radiology 289:293-312. https://doi.org/10.1148/radiol.2018172656

11. Schlomka JP, Roess E, Dorscheid R, et al (2008) Experimental feasibility of multi-energy photon-counting K-edge imaging in pre-clinical computed tomography. Phys Med Biol 53:4031-4047. https://doi.org/10.1088/0031-91 55/53/15/002

12. Riederer I, Si-Mohamed S, Ehn S, et al (2019) Differentiation between blood and iodine in a bovine brain -- Initial experience with spectral photoncounting computed tomography (SPCCT). PLoS One 14:e0212679. https:// doi.org/10.1371/journal.pone.0212679

13. Si-Mohamed S, Cormode DP, Bar-Ness D, et al (2017) Evaluation of spectral photon counting computed tomography K-edge imaging for determination of gold nanoparticle biodistribution in vivo. Nanoscale 9:18246-18257. https://doi.org/10.1039/c7nr01153a

14. Si-Mohamed S, Tatard-Leitman V, Laugerette A, et al (2019) Spectral photoncounting computed tomography (SPCCT): in-vivo single-acquisition multiphase liver imaging with a dual contrast agent protocol. Sci Rep 9:8458. https://doi.org/10.1038/s41598-019-44821-z

15. Ding H, Zhao B, Baturin P, Behroozi F, Molloi S (2014) Breast tissue decomposition with spectral distortion correction: A postmortem study. Med Phys 41:101901. https://doi.org/10.1118/1.4894724

16. Kirkbride TE, Raja AY, Müller K, Bateman CJ, Becce F, Anderson NG (2017) Discrimination between calcium hydroxyapatite and calcium oxalate using multienergy spectral photon-counting CT. AJR Am J Roentgenol 209:10881092. https://doi.org/10.2214/AJR.17.18394

17. Stamp LK, Anderson NG, Becce F et al (2019) Clinical utility of multi-energy spectral photon-counting computed tomography in crystal arthritis. Arthritis Rheumatol 71:1158-1162. https://doi.org/10.1002/art.40848

18. Große Hokamp N, Gilkeson R, Jordan MK, et al (2019) Virtual monoenergetic images from spectral detector $C T$ as a surrogate for conventional $C T$ images: Unaltered attenuation characteristics with reduced image noise. Eur J Radiol 117:49-55. https://doi.org/10.1016/j.ejrad.2019.05.019

19. Rajendran K, Löbker C, Schon BS, et al (2017) Quantitative imaging of excised osteoarthritic cartilage using spectral CT. Eur Radiol 27:384-392. https://doi.org/10.1007/s00330-016-4374-7

20. Roemer F, Eckstein F, Hayashi D, Guermazi A (2014) The role of imaging in osteoarthritis. Best Pract Res Clin Rheumatol 28:31-60. https://doi.org/10.101 6/j.berh.2014.02.002

21. Steadman R, Herrmann C, Livne A (2017) ChromAIX2: A large area, high count-rate energy-resolving photon counting ASIC for a Spectral CT Prototype. Nucl Instrum Methods Phys Res Sect Accel Spectrometers Detect Assoc Equip 862:18-24. https://doi.org/10.1016/j. nima.2017.05.010

22. Kellgren JH, Lawrence JS (1957) Radiological assessment of osteoarthritis. Ann Rheumat Dis 16:494-502. https://doi.org/10.1136/ard.16.4.494

23. Kalender WA (2014) Dose in X-ray computed tomography. Phys Med Biol 59:R129-R150. https://doi.org/10.1088/0031-9155/59/3/R129
24. Aslund M, Fredenberg E, Telman M, Danielsson M (2010) Detectors for the future of X-ray imaging. Radiat Prot Dosimetry 139:327-333. https://doi. org/10.1093/rpd/ncq074

25. McErlain DD, Milner JS, Ivanov TG, Jencikova-Celerin L, Pollmann SI, Holdsworth DW (2011) Subchondral cysts create increased intra-osseous stress in early knee OA: a finite element analysis using simulated lesions. Bone 48:639-646. https://doi.org/10.1016/j.bone.2010.11.010

26. Burnett WD, Kontulainen SA, McLennan CE, Hunter DJ, Wilson DR, Johnston JD (2014) Regional depth-specific subchondral bone density measures in osteoarthritic and normal patellae: in vivo precision and preliminary comparisons. Osteoporos Int 25:1107-1114. https://doi.org/10.1007/s00198013-2568-2

27. Gao L, Cucchiarini M, Madry H (2020) Cyst formation in the subchondral bone following cartilage repair. Clin Transl Med 10:e248. Review. https://doi. org/10.1002/ctm2.248

28. Kroker A, Zhu Y, Manske SL, Barber R, Mohtadi N, Boyd SK (2017) Quantitative in vivo assessment of bone microarchitecture in the human knee using HR-pQCT. Bone 97:43-48. https://doi.org/10.1016/j.bone.2016.12. 015

29. Bornefalk H (2012) XCOM intrinsic dimensionality for low-Z elements at diagnostic energies. Med Phys 39:654-657. https://doi.org/10.1118/1.36753 99

30. Ducros N, Abascal J, Sixou B, Rit S, Peyrin F (2017) Regularization of nonlinear decomposition of spectral X-ray projection images. Med Phys 44: e174-e187. https://doi.org/10.1002/mp.12283

31. Kim K, Ye JC, Worstell W et al (2015) Sparse-view spectral CT reconstruction using spectral patch-based low-rank penalty. IEEE Trans Med Imaging 34: 748-760. https://doi.org/10.1109/TMI.2014.2380993

32. Semerci O, Hao N, Kilmer ME, Miller EL (2014) Tensor-based formulation and nuclear norm regularization for multienergy computed tomography. Trans Image Process IEEE 23:1678-1693. https://doi.org/10.1109/TIP.2014.2305840

33. Touch M, Clark DP, Barber W, Badea CT (2016) A neural network-based method for spectral distortion correction in photon counting X-ray $C T$. Phys Med Biol 61:6132-6153. https://doi.org/10.1088/0031-9155/61/16/6132

34. Arridge S, Maass P, Öktem O, Schönlieb C-B (2019) Solving inverse problems using data-driven models. Acta Numerica 28:1-174. https://doi.org/10.1017/ S0962492919000059

35. Bussod S, Abascal JFPJ, Ducros N et al (2019) In: Human knee phantom for spectral CT: validation of a material decomposition algorithm (ed) 2019 IEEE 16th International Symposium on Biomedical Imaging (ISBI 2019), pp 1170 1173. https://doi.org/10.1109//SBI.2019.8759192

36. Ravishankar S, Ye JC, Fessler JA (2020) Image reconstruction: from sparsity to data-adaptive methods and machine learning. Proc IEEE Inst Electr Electron Eng 108:86-109. https://doi.org/10.1109/JPROC.2019.2936204

37. Abascal JFPJ, Si-Mohamed S, Douek P, Chappard C, Peyrin F (2019) In: A sparse and prior based method for 3D image denoising (ed) 2019 27th European Signal Processing Conference (EUSIPCO), pp 1-5. https://doi.org/1 0.23919/EUSIPCO.2019.8902564

\section{Publisher's Note}

Springer Nature remains neutral with regard to jurisdictional claims in published maps and institutional affiliations.

\section{Submit your manuscript to a SpringerOpen ${ }^{\circ}$ journal and benefit from:}

- Convenient online submission

- Rigorous peer review

- Open access: articles freely available online

- High visibility within the field

- Retaining the copyright to your article

Submit your next manuscript at $>$ springeropen.com 